\title{
MeasureIce: Accessible on-the-fly measurement of ice thickness in cryo-electron microscopy
}

Hamish G. Brown ${ }^{* 1}$ and Eric Hanssen ${ }^{1,2}$

1 lan Holmes Imaging Center, Bio21 Molecular Science \& Biotechnology Institute, University of Melbourne, Parkville, Victoria, Australia.

2 Department of Biochemistry and Molecular Biology, The University of Melbourne, Parkville, Victoria, Australia.

*Corresponding author hgbrown@unimelb.edu.au

\section{Abstract}

Ice thickness is arguably one of the most important factors limiting the resolution of protein structures determined by cryo electron microscopy. The amorphous atomic structure of the ice that stabilizes and protects biological samples in cryo-EM grids also imprints some addition noise in the TEM images. Ice that is too thick jeopardizes the success of particle picking and reconstruction of the biomolecule in the worst case and, at best, deteriorates eventual map resolution. Minimizing the thickness of the ice layer and thus the magnitude of its noise contribution is thus imperative in cryoEM grid preparation. In this paper we introduce Measurelce, a simple, easy to use ice thickness measurement tool for screening and selecting acquisition areas of cryo-EM grids. We show that it is possible to simulate thickness-image intensity look-up tables using elementary scattering physics and thereby adapt the tool to any microscope without time consuming experimental calibration. We benchmark our approach using two alternative techniques: the "ice-channel" technique and tiltseries tomography. We also demonstrate the utility of ice thickness measurement for selecting holes in gold grids containing an Equine apoferritin sample, achieving a 1.88 Ångstrom resolution in subsequent refinement of the atomic map.

\section{Introduction}

Single particle cryogenic transmission electron microscopy (cryo-EM) is a characterization technique for biological macromolecules that has seen rapid growth in the past decade, both in the number of structures solved and resolutions of the reconstructed 3D molecular models ${ }^{1}$. Sub-3 A resolutions which allow the fitting of amino acid side chains in the three-dimensional density map ${ }^{2}-$ are now common and the current resolution record is just above $1.2 \AA^{3}$. The history of the development of cryo-EM has principally been one of surmounting the inherent challenges of signal-to-noise.

Biological macromolecules are susceptible to electron beam radiolysis, the breaking of chemical bonds by the high-energy electron beam of the TEM instrument ${ }^{4}$. Vitrification, plunge-freezing of grids so that the water freezes into amorphous (vitreous) ice, provides some protection by reducing the diffusion of radiolysis products so permits stability of at least the low resolution features of a protein up to an electron dose of around $40-70 \mathrm{e} / \AA^{2}{ }^{5}$. Even with this dose level cryo-EM images are dominated by Poisson counting or "shot" noise, the quantum-statistical fluctuations in the numbers of electrons arriving at any given camera pixel during acquisition, overwhelming the atomic resolution structure that would be otherwise evident in single images. In the single particle analysis 
(a) Electron tomography

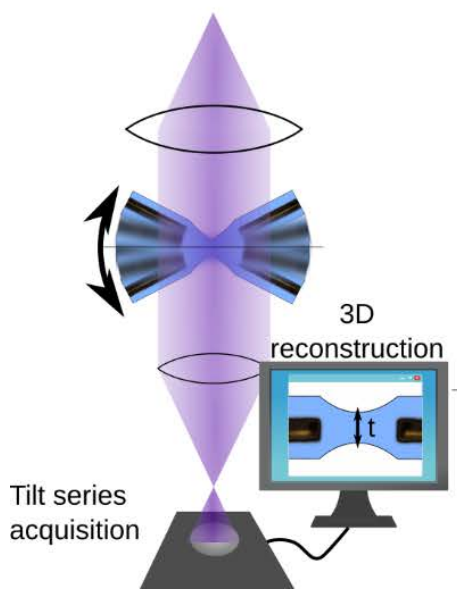

(b) Ice-channel method

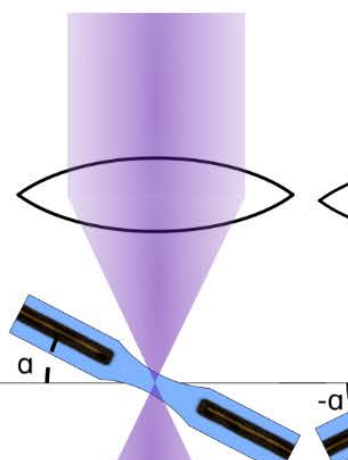

Hole burnt in specimen at $+a$
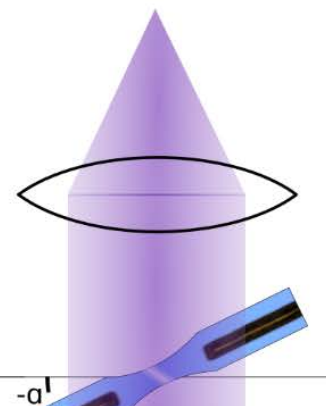

Ice thickness inferred from image measured at $-a$ (c) Energy-filter method

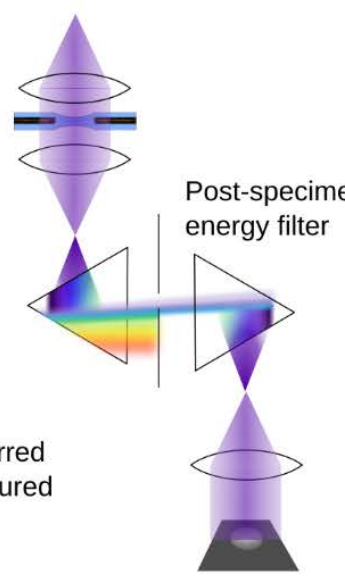

(d) Aperture limited scattering (ALS)

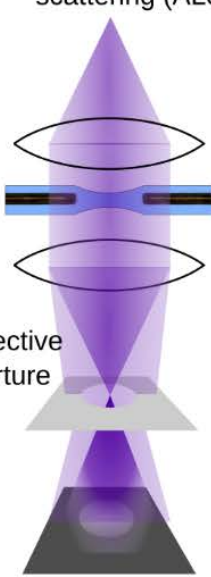

Figure 1 Methods for measuring ice thickness in cryo-TEM, see body text for more detail.

(SPA) workflow, sophisticated Bayesian algorithms combine tens or even hundreds of thousands of images into a single three-dimensional reconstruction such that the available signal then becomes sufficient to finally reach the resolutions approaching what the TEM is inherently capable of ${ }^{6}$.

Since signal to noise is inherently limited in cryo-EM yet critical to successfully solving structures via the technique, much research effort is applied to reducing additional sources of noise. The most striking example is the recent development of direct electron detectors which feature a significantly improved detector quantum efficiency (DQE), a measure of the additional noise the detection system adds to the measured micrograph ${ }^{7}$. The disordered atomic structure of the vitreous ice that proteins are necessarily encapsulated within in cryo-EM is itself an additional source of noise ${ }^{8}$ and thicker ice means noisier images. Therefore, a key step in the cryo-EM single particle analysis (SPA) workflow is the inspection of samples on an economical screening TEM to ensure that sufficient densities of particles can be found in thin ice regions of the grid before these samples advance to data collection on expensive high end acquisition instruments and consume much needed computational resources. This is hampered by the fact that most of these methods require either time-consuming post-experiment analysis or pre-experimental calibration so are difficult to easily incorporate in screening workflows. Commonly used methods are summarized in Fig. 1 and include tomography, the so-called "ice-channel" method, the use of an energy filter or the aperture-limited scattering (ALS) approaches. Tomography, Fig. 1(a), has the advantage of providing a maximal amount of information by reconstructing the three-dimensional distribution of protein, including its interaction with the air-water interface, in the ice layer ${ }^{9}$. The longer acquisition and data processing time means it is unsuited to on-the-fly analysis. The ice-channel method, Fig. 1(b), involves condensing the electron beam to burn a hole through the ice in one stage tilt (eg. -30 degrees) and then tilting the stage to a second orientation (eg. +30 degrees) from which the thickness of the icelayer can be inferred by the projected length of the ice-channel in a recorded image ${ }^{10-12}$. In the energy filter approach, Fig. 1(c), comparison of the intensities of an un-filtered image and one where a post-specimen energy filter removes electrons that have been inelastically scattered by the ice layer allows for quick on-the-fly measurement of ice thickness ${ }^{10,11}$. The ALS approach, Fig. 1(d), uses a post-specimen objective aperture to remove electrons scattered both elastically and inelastically by the ice meaning thicker-ice regions of the grid appear darker in the image ${ }^{13}$. We focus on the ALS method in this work since, once calibrated, ice thickness is measured directly from single 
micrographs meaning the method is fast, only requires the most basic of screening microscopes without an energy filter or tomography software and minimal user training. Additionally, the ALS method is best used at low magnifications where the beam is typically spread so wide that the intensity is much less than $1 \mathrm{e} / \AA^{2}$. Large regions of the grid can be surveyed with minimal damage to the specimen, making this an appropriate technique for selecting holes for acquisition, unlike tomography (which requires higher magnification and dose) and the ice-channel method which is necessarily destructive to the region of interest.

For ALS images recorded in-focus (so that defocus induced phase contrast is minimized) the intensity for a region of amorphous ice is well approximated by ${ }^{13}$

$$
\mathrm{I}=\mathrm{I}_{0} \mathrm{e}^{-\mathrm{t} / \mathrm{T}_{\mathrm{eff}},}
$$

Where $I_{0}$ is the incident intensity of the electron beam (that measured in the absence of any sample in the path of the beam), $t$ is the thickness of the ice and $T_{\text {eff }}$ is the effective mean-free-path for elastic and inelastic scattering of an electron to an angle large enough that the objective aperture will remove it from the image. This effective mean-free-path, $T_{\text {eff, }}$ is dependent on a complex interplay between elastic and inelastic scattering which we will review in the methods section of this paper and for a well-aligned microscope is a function of the accelerating voltage, which determines the degree of elastic and inelastic scattering for a given ice thickness, and aperture size,which determines the scattering angle beyond which scattered electrons will be removed from the image. Note that the relevant value of the aperture size is in units of inverse length or angle (ie. mrad) measured using a diffraction standard such as gold nanoparticles, not the aperture physical size in micron values typically provided by the instrument manufacturer. In previous work $T_{\text {eff }}$ was measured by extensive experimental calibration ${ }^{11,13}$, in this work we demonstrate how $T_{\text {eff }}$ can be estimated through first principles simulation and thus the method be applied to a TEM without the need for the time-consuming calibration step. We benchmark this approach using the ice-channel and tomography ice thickness measurements and present a software package named Measurelce that allows for ice thickness measurements using ALS during data collection. Finally we demonstrate the utility of quantitative ice thickness measurements pre-acquisition via a $1.88 \AA$ reconstruction of equine apoferritin, which is a $0.22 \AA$ improvement on previously reported results ${ }^{14,15}$. We refer to our technique of measuring ice thickness using aperture limited scattering informed by simulated look-up tables as the "Measurelce approach".

\section{Results}

\section{Comparison with the ice channel approach}

Simulated ice thickness-image intensity curves were simulated for three different microscopes, a ThermoFisher Talos L120C, Talos Arctica G2 and Titan Krios G4, operated at different electron beam energies, respectively $120 \mathrm{keV}, 200 \mathrm{keV}$ and $300 \mathrm{keV}$, and using different apertures. These curves are plotted along with experimental benchmarks using the ice-channel method to measure ice 

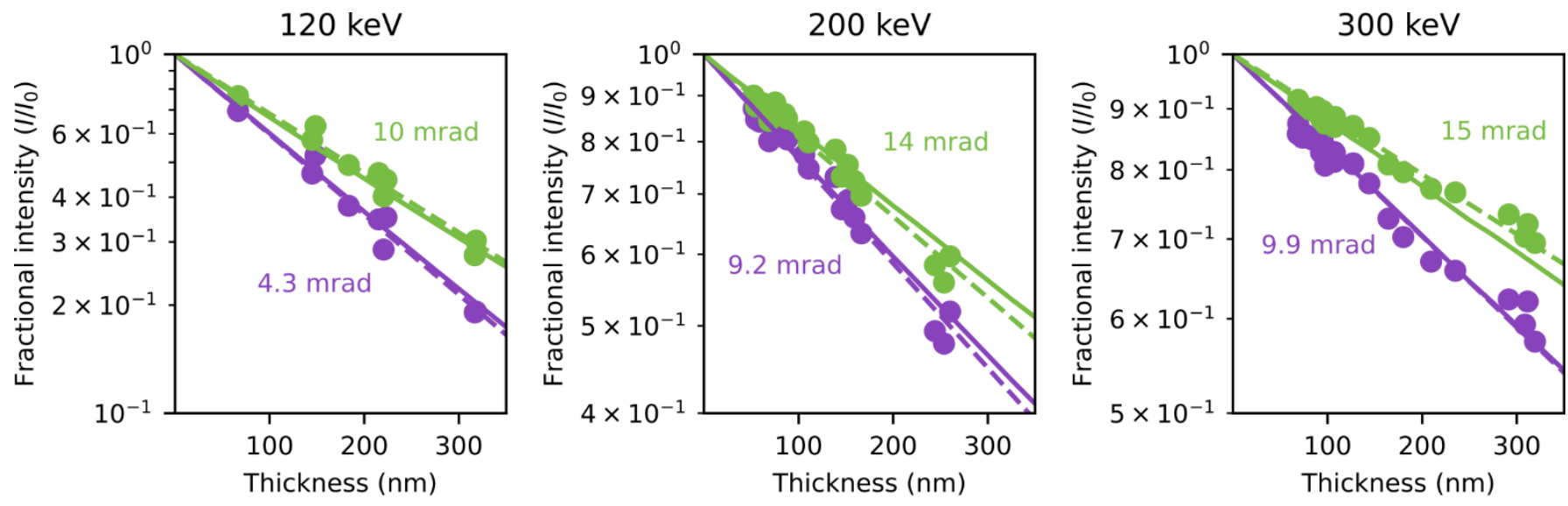

Experiment $\bullet / \odot$ Experiment line of best fit $---/---$ Measurelce simulation $\square / \square$

Figure 2 Simulated Ice thickness - image intensity maps (Measurelce simulation) for three different microscope accelerating voltages and different objective apertures with benchmarking using the ice channel method (Experiment) along with the line of best

thicknesses in regions of vitrified cryo-EM in Fig. 2. Good agreement between experiment and theory was found for all experimental accelerating voltages suggesting that the simulations correctly incorporate all of the important and necessary physics for the energy ranges typically used in cryoEM and can be relied on to produce accurate ice thickness maps.

Comparisons with tomography

By way of an additional benchmark we compared ice thickness measurements from the Measurelce approach to estimates from tomography. Since significant information loss in the $z$ direction resulting from the missing wedge in a standard tilt series acquisition makes it difficult to detect the top and bottom surfaces of the ice in a pure water sample, a $7 \mathrm{mg} / \mathrm{ml}$ apoferritin sample was used for this experiment. Apo-ferritin particles were used as an indicator of the extent of the water volume.

Four tomograms were acquired using a ThermoFisher Talos Arctica, operated at $200 \mathrm{keV}$, for different holes in the grid, selected using Measurelce to give a spectrum of ice thicknesses up to about $100 \mathrm{~nm}$. We then used a custom script to fit a surface function to these points estimate the profile of the ice layer, this is shown for a representative Quantifoil ${ }^{\circledR}$ hole in Fig. $3 a$. The ice thickness profile was then calculated as the height difference between the top and bottom layers of the ice. A comparison of Measurelce and tomography estimates of ice thickness for all the holes is shown in (Fig 3c) and reasonable agreement is found between the two approaches. A Measurelce thickness map incorporating two of the tomograms ( 1 \& 2 or blue and red) is inset in (Fig 3d). for reference.

Since de-ionized water was used in the ice-channel measurements, using an apo-ferritin sample for this benchmark had the additional benefit of testing whether the presence of a standard cryo-EM 
bioRxiv preprint doi: https://doi.org/10.1101/2021.12.08.471523; this version posted December 9,2021 . The copyright holder for this preprint (which was not certified by peer review) is the author/funder, who has granted bioRxiv a license to display the preprint in perpetuity. It is made available under aCC-BY 4.0 International license.

(a) Tomography

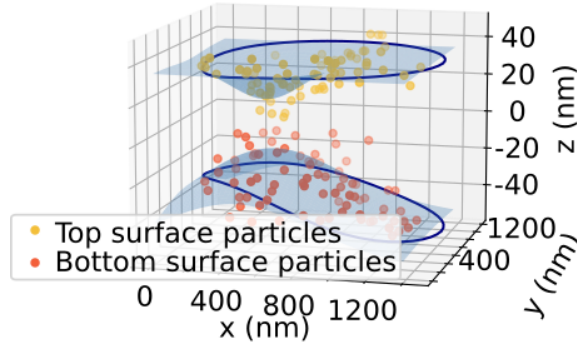

(b) Single hole thickness maps

Measurelce Tomography

\section{$0.5 \mu \mathrm{m}$}

$0 \quad 20 \quad 40 \quad 60 \quad 80 \quad 100$

Ice thickness $(\mathrm{nm})$ (c) Measurelce tomography comparision

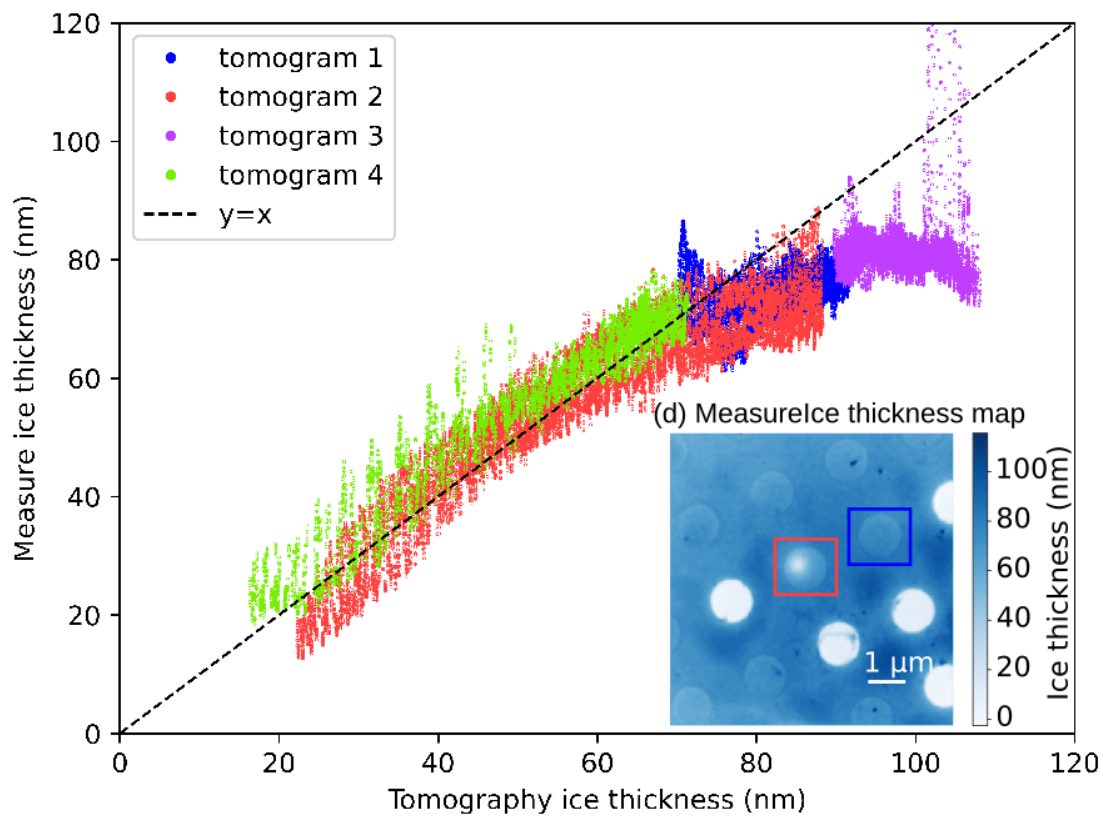

Figure 3 Benchmarking of Measureice with electron tomography. For each hole particles at the extremities in z were manually picked from the grid and a surface fitted to these points (a), the height difference between these two surfaces is taken to be the estimated thickness and this is plotted in (b) alongside the same grid hole measured using the Measurelce approach. Shown in (c) is a comparison of ice thickness as measured by tomography and measureice for four different grid holes on an apo-ferritin containing carbon quantifoil grid, the measureice thickness map for two of the tomograms is inset in (d).

protein sample, both protein and buffer, would affect the accuracy of ice thickness measurements. Protein typically has a shorter inelastic mean-free path than the surrounding water so the presence of protein in significant quantities will mean the solution has a slightly shorter inelastic mean-free path on average and Measurelce might be expected underestimate thickness. This effect is not observed in our experiments which gives confidence in using the Measurelce approach for standard cryo-EM samples, though caution might be advised with samples containing large amounts of additives such as glycerol, or with a concentrated salt buffer.

\section{Uncertainty in literature values of inelastic mean free path}

The simulated ice thickness-image intensity lookup tables depend on the energy dependent mean free path for inelastic scattering, $T$, which is taken from recent measurements by Yesibolati et al. ${ }^{16}$ for $120 \mathrm{kV}$ and $300 \mathrm{kV}$. For other values electron energy take the following equation to approximate the mean free path ${ }^{17,18}$ and use that equation as a basis for interpolation between measured values of $\mathrm{T}^{16}$

$$
T=\frac{M_{W} \beta^{2}}{9.03 \times 10^{-10} \rho Z^{1 / 2} \ln \frac{\beta^{2}\left(U_{0}+m c^{2}\right)}{10}}
$$


(a)

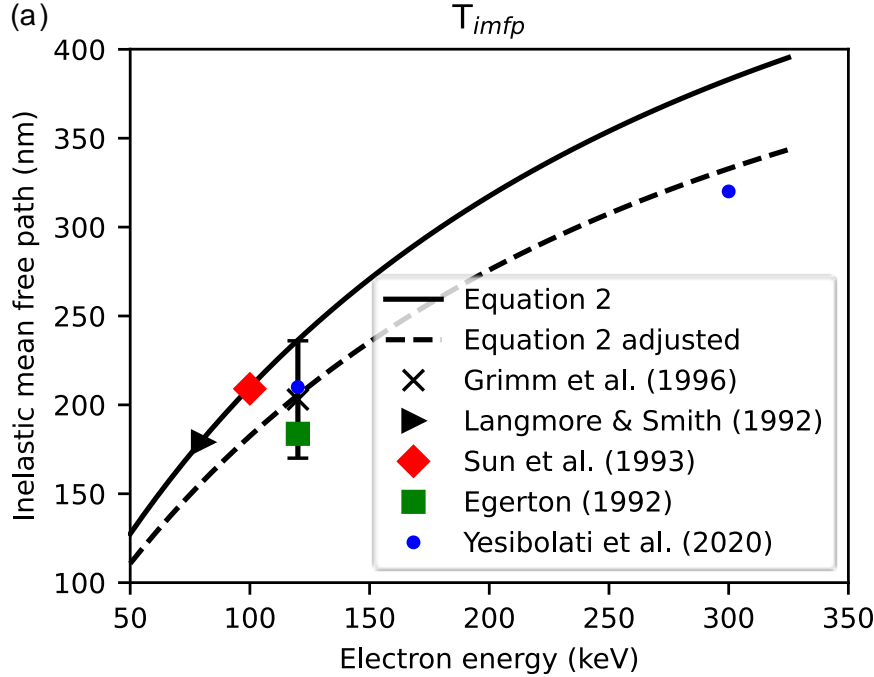

(b)

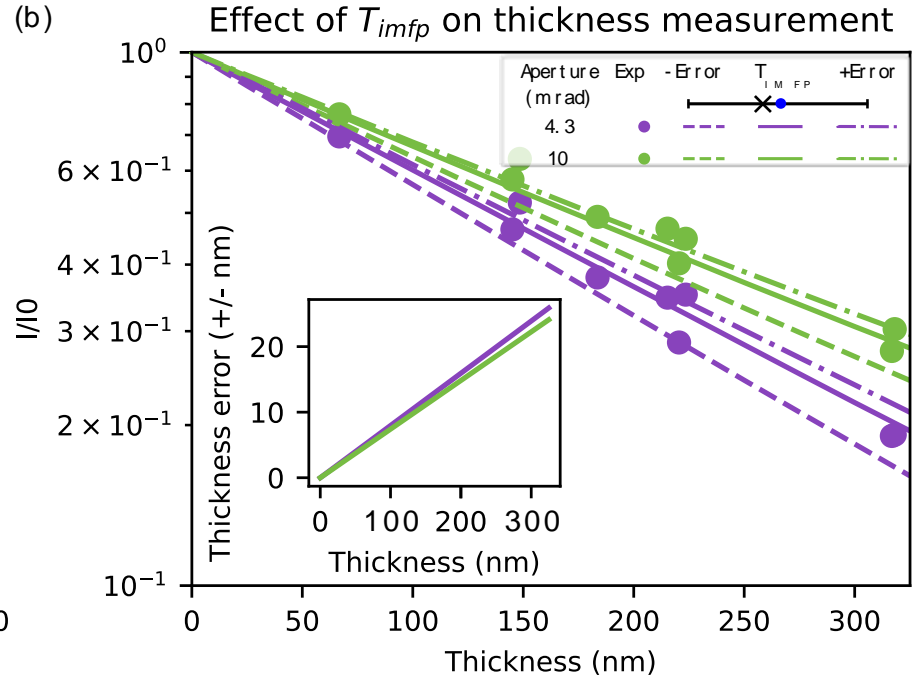

Figure 4 (a) Literature values for experimental mean free path plotted alongside equation 2, and the interpolating function for mean free paths used in Measurelce (equation 2 adjusted). (b) Ice thickness measurement errors that would be implied by the effect of experimental errors from Grimm et al. ${ }^{19}$. Solid line is the calibration curve implied by Yesibolati's mean free path measurement at $120 \mathrm{kV}^{16}$ and the dashed lines represent the extremities of the error bars in (a), the plot instead is the error as a function of thickness implied by Yesibolati's measurements..

Experimentally measured values for the inelastic mean free path ${ }^{16,18-21}$ and those predicted by equation 2 and our fit of equation (2) to the results of Yesibolati et al. ${ }^{16}$ (labelled "Equation 2 adjusted"] are plotted in Fig. 4. We consider the result of the experiment of Yesibolati et al. ${ }^{16}$ the most rigorous to date since mean free path is directly measured from electron energy-loss spectroscopy (EELS) data recorded for an electron probe scanned across water channels of known geometry. However it must be acknowledged that the inputted value of inelastic mean-free path is the most obvious source of error in the Measurelce calculations. Grimm et al. ${ }^{19}$, whose value for the inelastic mean free path of an electron at $120 \mathrm{kV}$ agrees well with Yesibolati et al. ${ }^{16}$, conducted a rigorous error analysis with their experiment and this presents an opportunity to investigate the effect of errors in inelastic mean free path. Fig. 4(b) is the same as Fig 2(a) which compares Measurelce simulations with benchmark ice thickness measurements. Additional Measurelce calibration curves, generated with the inelastic mean free path changed to the extremities of Grimm et al.'s error bars, have been plotted in dashed lines. Inset in this figure is a plot of the implied thickness error for both the $\mathrm{L} 120 \mathrm{C}$ apertures as a function of nominal thickness (ie. Implied by Yesibolati's value of the inelastic mean free path). It can be seen that this implied ice thickness error is consistently well below $10 \%$, sufficient for estimating ice thickness in cryo-EM.

\section{MeasureIce, a software tool for measuring ice thickness}

Once ice thickness image intensity look-up tables are simulated for a microscope's given apertureaccelerating voltage combination this information can be used to infer ice thickness on the fly. We have developed a Python software tool to facilitate thickness measurements using these simulated look-up tables using the pyqtgraph (https://www.pyqtgraph.org/) graphics and user interface Python library. The source code for the Measurelce python script is available on GitHub (https://github.com/HamishGBrown/Measurelce). It can be deployed using a python interpreter 
bioRxiv preprint doi: https://doi.org/10.1101/2021.12.08.471523; this version posted December 9, 2021. The copyright holder for this preprint (which was not certified by peer review) is the author/funder, who has granted bioRxiv a license to display the preprint in perpetuity. It is made available under aCC-BY 4.0 International license.

installed on the microscope control or support PC or compiled into a stand-alone Windows executable using the py_installer (https://www.pyinstaller.org/) package.

A screenshot of the graphical user interface (GUI) is shown in Fig. 5. The user can select between different pre-computed image intensity-thickness calibrations (which are stored as hdf5 files, https://www.hdfgroup.org/HDF5/), for example for different microscopes or for different accelerating voltages on the same microscope, from the drop-down menu at the bottom left. Raw images are loaded as tif, ser (using the openNCEM, https://github.com/ercius/openNCEM, package developed by Peter Ercius at Lawrence Berkeley National Lab) or mrc (using the Python mrcfile module ${ }^{22}$ ) files and displayed on the left-hand side of the interface. The vacuum intensity $I_{0}$, the image intensity recorded for regions of the image with no material in the path of the beam, can be selected by moving with the mouse pointer the red line superimposed on the intensity histogram immediately to the right of the raw image. Once $I_{0}$ has been set, the user generates an ice thickness map by pressing the "Generate Ice thickness map" button. Ice thickness measurements can be read off the interface by hovering the mouse pointer over the region of interest. The ice thickness map can be saved as a 32bit tiff file for reference and further analysis or as a matplotlib ${ }^{23}$ plot in png or pdf format by clicking the "save ice thickness map" button. More detailed step-by-step instructions for Measurelce may be found in the Extended Data section.

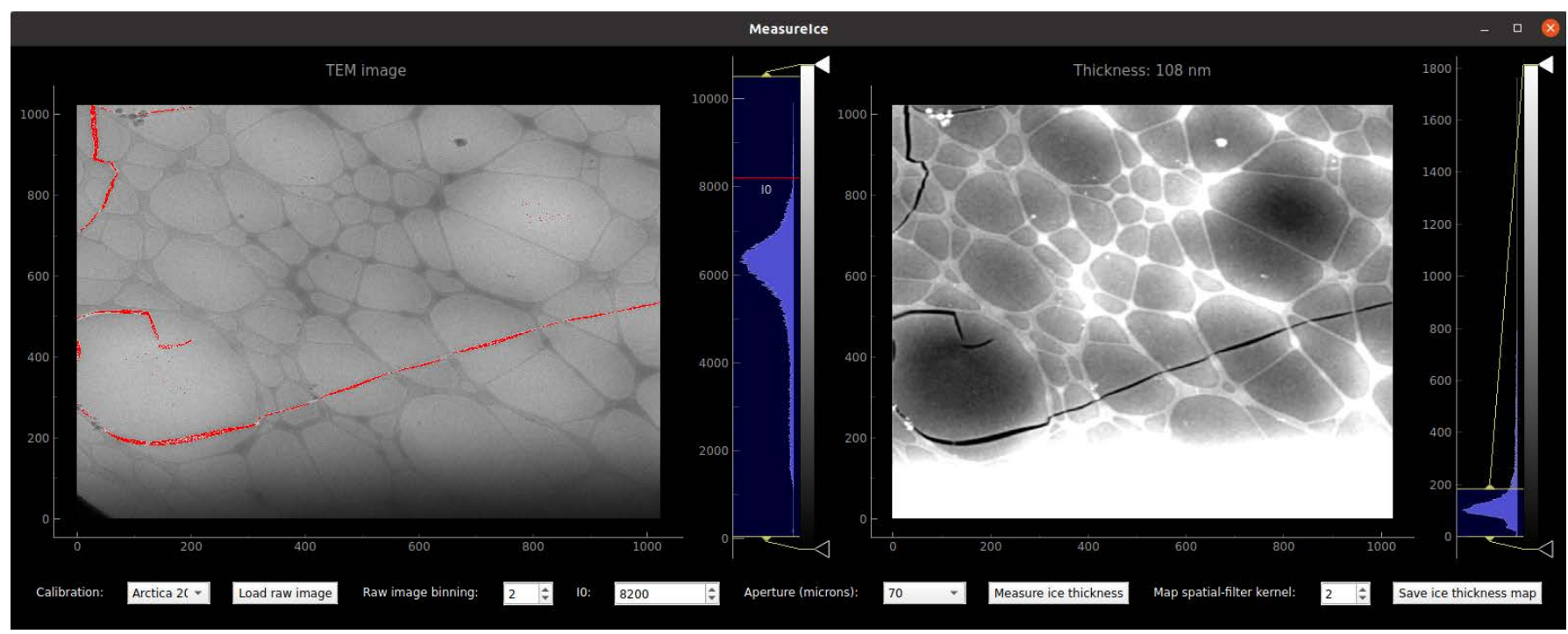

Figure 5 Screenshot of the Measurelce software tool. In the left panel a raw TEM image is loaded, 10, the incident intensity of the electron beam is set using the red line on the histogram or using the input on the bottom of the interface. The ice thickness map is displayed in the right panel of the interface.

\section{Using MeasureIce for screening and data collection}

The ultimate goal of Measurelce is to ensure that cryo-EM practitioners can more rapidly and quantitatively achieve minimum possible ice thicknesses for a given sample, which in turn reduces the effective signal to noise of micrographs resulting in higher resolution reconstructions, potentially from fewer total particles. To this end Measurelce was evaluated on a standard cryo-EM single particle workflow with Equine Apoferritin (Sigma) vitrified on UltrAuFoil ${ }^{\circledR}$ gold grids. 
bioRxiv preprint doi: https://doi.org/10.1101/2021.12.08.471523; this version posted December 9, 2021. The copyright holder for this preprint (which was not certified by peer review) is the author/funder, who has granted bioRxiv a license to display the preprint in perpetuity. It is made available under aCC-BY 4.0 International license.

Compared to carbon Quantifoils ${ }^{\circledR}$ where ice thickness gradients are visible even on the carbon substrate and give some qualitative indication of ice thickness across a grid square, gold grids can be challenging for qualitatively evaluating ice thickness for inexperienced users. This is because the gold foil scatters the electron beam strongly and Bragg scattering by the polycrystalline foil dominates TEM images outside the grid holes, rendering the subtle ice thickness gradients invisible. Using Measurelce on the microscope support PC we were able to identify the relative intensity that corresponded to ice holes having $<15 \mathrm{~nm}$ thickness and select these holes for acquisition using the "Ice filter" setting on the EPU automated acquisition software of our Titan Krios - which selects holes for acquisition based on the mean image intensity inside the hole. Data processing was performed with Relion ${ }^{6}$ and Cryosparc $^{24}$ resulted in a $1.88 \AA$ map. This exceeds the current record resolution $2.1 \AA$ map of the same protein ${ }^{14,15}$. The reconstructed map is shown overlaid with the fitted molecular model $6 \mathrm{RJH}$ from ${ }^{14}$ in Fig. 6a with a representative micrograph shown in Fig. 6(b). The gold-standard Fourier shell correlation curve (GSFSC) is shown in Fig. 6(c) along with a rendering of the total EM map. Given differences in microscopes, cameras, grids and reconstruction workflows, we do not seek to attribute wholly this improvement to quantitative ice thickness curation. This result does however make the case for including quantitative ice thickness tools like Measurelce within cryo-EM screening and acquisition workflows, especially considering the convenience of the Measurelce software tool.
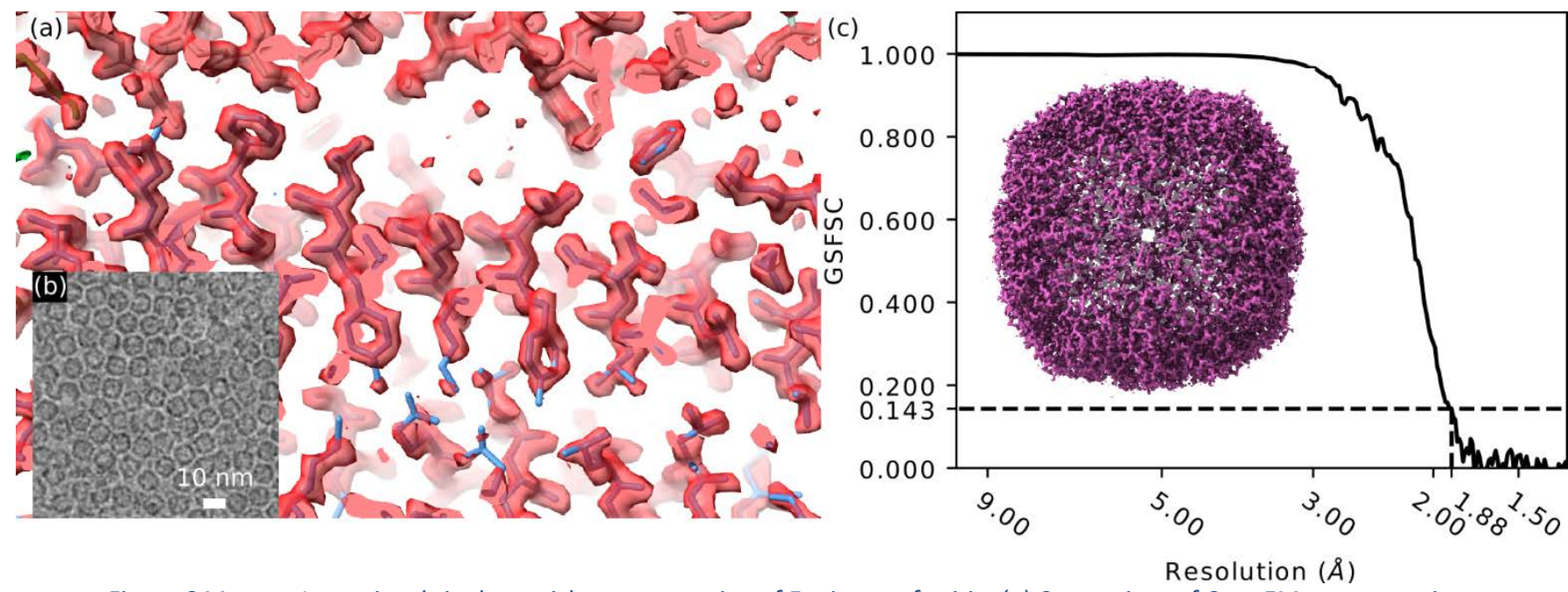

Figure 6 Measurelce assisted single particle reconstruction of Equine apoferritin. (a) Comparison of Cryo-EM reconstruction with structure model from ${ }^{14}$, (b) gold-standard Fourier shell correlation (GSFSC) assessment of map resolution and (c) rendering of the reconstructed molecular map.

\section{Conclusions}

The aperture limited scattering (ALS) approach provides a fast and convenient way to measure ice thickness on the fly, though till now the time-consuming nature of calibration means that the method is not as widespread in TEM as might ideally be the case. In this paper we have presented an approach to calculate look-up tables to convert image intensities in the ALS approach to icethickness values that allows the time-consuming process of experimental calibration to be skipped. This approach has been packaged for user convenience in software which we have named Measurelce meaning that barriers to including quantitative ice thickness measurements are substantially reduced. Thickness measurements compare favorably with alternative ice thickness measurement techniques and we have demonstrated the advantages of quantitative ice thickness measurements in general with a 1.88 Å resolution reconstruction of Equine apoferritin. 


\section{Methods}

\section{Simulating ice thickness maps}

In simulations we found that it was important to include both elastic and elastic scattering and the realistic structure of amorphous ice. A $10 \mathrm{~nm}^{3}$ cube of vitreous ice was generated using the GROMACS molecular dynamics simulation package ${ }^{25}$ according to the method detailed in Souza Junior et al. ${ }^{26}$. The multislice algorithm has been used a number of times to simulate electron elastic scattering in the cryo-EM context ${ }^{17,27,28}$ and here simulations were performed using the open source py_multislice package ${ }^{29}$. To incorporate multiple inelastic scattering we adapted the approach suggested by Egerton $^{30}$. For a given thickness $t$ the probability of an electron being inelastically scattered $n$ times due to plasmon excitation is given by,

$$
P_{n}(t)=\frac{I_{n}(t)}{I_{t}}=\frac{1}{n !}\left(\frac{t}{T}\right)^{n} e^{\frac{-t}{T}}
$$

The differential cross section for inelastic scattering to angle $\theta=\tan ^{-1}(\lambda k)$, where $k$ is the diffraction plane coordinate in units of inverse length and $\lambda$ is the electron wavelength, is parameterized as

$$
\frac{d \sigma}{d \theta}=\frac{1}{\theta^{2}+\theta_{E}^{2}}
$$

where $\theta_{E}$ is the energy-dependent characteristic scattering angle for plasmon excitation, this is provided by Yesibolat et al. ${ }^{16}$ for $120 \mathrm{kV}$ and $300 \mathrm{kV}$ and interpolated for other values. Since it is typically a fraction of mrad it tends to mainly determine the scattering distribution at small angles ( $<1 \mathrm{mrad}$ ), even doubling this figure in an ad-hoc way was not found to significantly affect the results of interest since most apertures are $>5 \mathrm{mrad}$. To incorporate plasmon inelastic scattering into simulation the electron wave function intensity resulting from the multislice simulations are convolved with the differential cross section in reciprocal space $n$ times for each instance of scattering and weighted with the probability that the electron will scatter that number of times from equation (2),

$$
|\Psi(k, t)|^{2}=P_{0}(t)\left|\Psi_{0}(k)\right|^{2}+P_{1}(t) \frac{d \sigma}{d \theta} *\left|\Psi_{0}(k)\right|^{2}+P_{2}(t) \frac{d \sigma}{d \theta} * \frac{d \sigma}{d \theta} *\left|\Psi_{0}(k)\right|^{2} \ldots
$$

The image intensity, as a fraction of incident beam flux $I_{0}$, for a given thickness of ice $t$ and acceptance angle $\alpha=\tan ^{-1}\left(\lambda k_{\alpha}\right)$ of the objective aperture, is then given by

$$
I(t) / I_{0}=\int_{0}^{k_{\alpha}}|\Psi(k, t)|^{2} d k
$$

Fractional image intensity as a function of ice thickness is calculated by the tool Generate_Measurelce_calibration.py and the user need only provide electron energy in keV and apertures in units of inverse Ångstrom or mrad to this utility. 
bioRxiv preprint doi: https://doi.org/10.1101/2021.12.08.471523; this version posted December 9, 2021. The copyright holder for this preprint (which was not certified by peer review) is the author/funder, who has granted bioRxiv a license to display the preprint in perpetuity. It is made available under aCC-BY 4.0 International license.

\section{Experimental benchmarking of the ice thickness look-up tables}

To confirm the validity of the simulation approach we benchmark it using the ice-channel approach. Here the sample is tilted to angle $\alpha$ and the beam is focused to a small spot, see Fig. 1(a), an image is recorded of the ice channel with the stage tilted to angle $\beta$ in the opposite direction. The thickness of the ice layer can be inferred from the size of the ice channel measured in projection,

$$
d=t \frac{\sin \alpha+\beta}{\cos \alpha} .
$$

It is most convenient to choose $\alpha=\beta$, ie. symmetric tilts, since then the projected size of the entrance and exit holes of the ice channel in the image will remain unchanged between the two acquisitions and the above equation simplifies to

$$
d=2 t \cdot \sin \alpha .
$$

The process is also considerably simplified by acquiring images at both tilts with a significant overfocus (around $20 \mu \mathrm{m}$ ) since this had the effect of high-pass filtering the images, making the edges of the ice channel entrance and exit holes more recognizable in the raw images.

\section{MeasureIce instructions}

1. Select the appropriate calibration file from the bottom left-most drop-down menu. These must be generated beforehand using the Generate_measureice_calibration.py utility.

2. Load a TEM raw image (mrc, tif or ser) using the "Load raw image" button. Users must be cautious that microscope software is not compressing the bit depth of images, for example saving with the tif format in ThermoFisher's TIA software compresses to the 8-bit or 16-bit integer datatype, since this will mean intensity measurements will not be comparable between acquisitions even if beam current and camera acquisition time is kept constant. To ensure accurate ice thickness measurements, users must be also careful that the image was recorded in focus (with minimum defocus induced phase contrast), with the beam in the parallel illumination condition and with the objective aperture aligned to be concentric with the microscope optic axis. The use of the "counting" mode for direct electron detectors such as the Gatan K2 and K3 or ThermoFisher Falcon cameras is discouraged since exceeding the recommended dose-per-pixel for accurate electron counting will mean a high rate of electron coincidence loss and that the detector response will not be linear. We have observed that the Measurelce software significantly underestimates ice thickness values when this happens. The low magnifications at ice thickness maps will normally be recorded (typically around 5000 times magnification) mean that the dose efficiency improvements resulting from electron counting are anyway irrelevant since the dose applied to the specimen should anyway be a fraction of an electron/ $\AA^{2}$.

3. Set the vacuum intensity $I_{0}$, the image intensity recorded for regions of the image with no material in the path of the beam, by moving with the mouse pointer the red line superimposed on the intensity histogram immediately to the right of the raw image. The Measurelce interface will identify regions of intensity greater than this value with a red mask overlayed on the image. This is shown for the case of a crack in the holey carbon film visible in the TEM image of Fig. 5. This approach is convenient for grids with holes in the ice but for images of grid regions with complete ice coverage $I_{0}$ needs to be measured from a separate 
bioRxiv preprint doi: https://doi.org/10.1101/2021.12 08.471523; this version posted December 9, 2021. The copyright holder for this preprint (which was not certified by peer review) is the author/funder, who has granted bioRxiv a license to display the preprint in perpetuity. It is made available under aCC-BY 4.0 International license.

image of an empty part of the grid using identical beam current and acquisition time and manually inputted into the $I_{0}$ section of the interface.

4. Select the aperture used to record the image from the drop-down menu in the centre of the bottom panel of the interface - Measurelce needs the aperture sizes in diffraction units of inverse Angstrom or mrad but the apertures can be labelled with the micron values that will actually appear in the microscope user interface.

5. Press the "Measure ice thickness" button. The ice thickness map will be shown in the righthand panel of the user interface and hovering the mouse above regions of the ice thickness map will cause the ice thickness for the region below the cursor to be displayed in the image title. Since there can be apparent large pixel-to-pixel variation in the ice thickness map, due mainly to Poisson counting noise, there is the option of filtering the image to remove much of this noise using a Gaussian low-pass filter. The amount of filtering is controlled by setting the filter kernel size in the "Map spatial-filter kernel" radio box.

6. Save the ice thickness map for future analysis and presentation by clicking the "Save ice thickness map" button.

\section{Grid preparation}

Equine Apoferritin at $7 \mathrm{mg} / \mathrm{ml}$ (Sigma, A3641) was prepared on 1.2/1.3 gold ultrafoil grids, glow discharged in a PELCO easiGlow system for 2 minutes with a $15 \mathrm{~mA}$ current, for single particle reconstructions and on 1.2/1.3 carbon quantifoil grids, glow discharged for 30 seconds with a $15 \mathrm{~mA}$ current, for tomography using a Leica EM GP2 automated plunge freezer. A $4 \mu \mathrm{L}$ volume was applied to the grids which were back-side blotted using the 'auto-blot' function of the EM GP2. The sample chamber was set to $22^{\circ}$ and $95 \%$ humidity.

\section{Tomography acquisition and reconstruction}

Images were recorded Talos Arctica (FEI) fitted with a Gatan K2 camera operated in electron counting mode and energy filtered with a $20 \mathrm{eV}$ slit inserted to improve contrast. Before tilt-series acquisition low magnification (5000x) images were recorded for later Measurelce analysis. The tomograms were reconstructed using IMOD ${ }^{31}$, with frames aligned via cross-correlation and binning of $4 \mathrm{~K}$ images by a factor 4 to enhance the contrast of the apo-ferritin particles. Particles at the extremities (in z) of the reconstructed volume were taken to indicate the upper and lower layers of ice and manually picked.

\section{Single particle analysis acquisition and reconstruction}

Grids were imaged on a Krios G4 cryoTEM (ThermoFisher) equipped with a Falcon 4 direct electron detector. The fractional intensity implied by Measurelce for ice thickness $<15 \mathrm{~nm}$ was used to select holes in the ThermoFisher EPU software using the "Ice filter" feature. The samples were imaged at a nominal magnification of 120,000 corresponding to a pixel size of $0.637 \AA$, calibrated using the 220 lattice reflection of a standard orientated gold specimen, with a defocus of $-0.4 \mu \mathrm{m}$ to $-1.0 \mu \mathrm{m}$. Exposure of $1.52 \mathrm{~s}$ were taken with The fluency was $5.34 \mathrm{e} / \mathrm{px} / \mathrm{s}$ resulting in a total dose of $20 \mathrm{e}^{-} / \AA^{2}$ on the sample with 8 frames per movie.

Reconstruction were carried out using a mixed workflow of RELION3.1 $1^{6,32}$ and Cryosparc3.0 ${ }^{24}$. Motion correction was carried out on gain-corrected movies with RELION3.1 own motion correction implementation. Motion corrected movies were imported in Cryosparc. CTF estimation was done using 
bioRxiv preprint doi: https://doi.org/10.1101/2021.12.08.471523; this version posted December 9,2021 . The copyright holder for this preprint (which was not certified by peer review) is the author/funder, who has granted bioRxiv a license to display the preprint in perpetuity. It is made available under aCC-BY 4.0 International license.

Patch CTF estimation. A total of 97,513 particles were extracted. Two rounds of 2D classification in Cryosparc resulted in a working dataset of 68,025 particles. Particles were imported in Relion using Pyem $^{33}$. Particles were used to create a de novo model then subjected to 3D refinement, CTF refinement and polishing follow by another round of CTF refinement. The ultimate 3D refinement resulted in a structure at $1.9 \AA$ with a B-factor of -66 . Ewald sphere correction was applied to the refinement results and improved the structure to $1.88 \AA$ and a B-factor of -54. Ewald sphere correction in Cryosparc v3.3.1 (not shown) also produced a structure with $1.89 \AA$ A resolution and a B-factor of -54 . The resolution was estimated using the gold standard FSC $=0.143$, calculated using a relaxed solvent map (deposition EMD-25619).

\section{Model fitting.}

Pdb structure $6 \mathrm{RJH}^{14}$ was fitted into the Coulomb potential map using rigid body fitting in Chimera$X^{34}$.

\section{Code availability}

The Python source code for Measurelce (https://github.com/HamishGBrown/Measurelce, https://doi.org/10.5281/zenodo.5751190) and py_multislice ${ }^{29}$ (https://github.com/HamishGBrown/py multislice, https://doi.org/10.5281/zenodo.5762736) are available via GitHub.

\section{References}

1. Electron Microscopy Data Bank (https://www.ebi.ac.uk/emdb/statistics; 2021).

2. Rosenthal, P.B. Testing the validity of single-particle maps at low and high resolution. Methods in enzymology 579, 227-253 (2016).

3. Nakane, T. et al. Single-particle cryo-EM at atomic resolution. Nature 587, 152-156 (2020).

4. Glaeser, R.M. Specimen behavior in the electron beam. Methods in enzymology 579, 19-50 (2016).

5. Grant, T. \& Grigorieff, N. Measuring the optimal exposure for single particle cryo-EM using a $2.6 \AA$ reconstruction of rotavirus VP6. elife 4, e06980 (2015).

6. Scheres, S.H.W. RELION: implementation of a Bayesian approach to cryo-EM structure determination. Journal of structural biology 180, 519-530 (2012).

7. McMullan, G., Faruqi, A.R. \& Henderson, R. Direct electron detectors. Methods in enzymology 579, 1-17 (2016).

8. Baxter, W.T., Grassucci, R.A., Gao, H. \& Frank, J. Determination of signal-to-noise ratios and spectral SNRs in cryo-EM low-dose imaging of molecules. Journal of structural biology 166, 126-132 (2009).

9. Noble, A.J. et al. Routine single particle CryoEM sample and grid characterization by tomography. Elife 7, e34257-e34257 (2018).

10. Feja, B. \& Aebi, U. Determination of the inelastic mean free path of electrons in vitrified ice layers for on-line thickness measurements by zero-loss imaging. Journal of microscopy 193, 15-19 (1999).

11. Cheng, A., Fellmann, D., Pulokas, J., Potter, C.S. \& Carragher, B. Does contamination buildup limit throughput for automated cryoEM? Journal of structural biology 154, 303-311 (2006).

12. Angert, I., Burmester, C., Dinges, C., Rose, H. \& Schröder, R.R. Elastic and inelastic scattering cross-sections of amorphous layers of carbon and vitrified ice. Ultramicroscopy 63, 181-192 (1996).

13. Rice, W.J. et al. Routine determination of ice thickness for cryo-EM grids. Journal of structural biology 204, 38-44 (2018).

14. Naydenova, K., Peet, M.J. \& Russo, C.J. Multifunctional graphene supports for electron cryomicroscopy. Proceedings of the National Academy of Sciences 116, 11718-11724 (2019). 
bioRxiv preprint doi: https://doi.org/10.1101/2021.12.08.471523; this version posted December 9, 2021. The copyright holder for this preprint (which was not certified by peer review) is the author/funder, who has granted bioRxiv a license to display the preprint in perpetuity. It is made available under aCC-BY 4.0 International license.

15. Kopylov, M. et al., Edn. 07/08/2019 (https://www.ebi.ac.uk/pdbe/entry/pdb/6pxm; 2019).

16. Yesibolati, M.N. et al. Electron inelastic mean free path in water. Nanoscale 12, 20649-20657 (2020).

17. Vulović, M. et al. Image formation modeling in cryo-electron microscopy. Journal of structural biology 183, 19-32 (2013).

18. Langmore, J.P. \& Smith, M.F. Quantitative energy-filtered electron microscopy of biological molecules in ice. Ultramicroscopy 46, 349-373 (1992).

19. Grimm, R., Typke, D., Bärmann, M. \& Baumeister, W. Determination of the inelastic mean free path in ice by examination of tilted vesicles and automated most probable loss imaging. Ultramicroscopy 63, 169-179 (1996).

20. Sun, S., Shi, S. \& Leapman, R. Water distributions of hydrated biological specimens by valence electron energy loss spectroscopy. Ultramicroscopy 50, 127-139 (1993).

21. Egerton, R. A data base for energy-loss cross-section and mean free path. Fiftieth Annual Proceedings of the Electron Microoscopy Sciety of America, 1264-1265 (1992).

22. Burnley, T., Palmer, C.M. \& Winn, M. Recent developments in the CCP-EM software suite. Acta Crystallographica Section D: Structural Biology 73, 469-477 (2017).

23. Hunter, J.D. Matplotlib: A 2D graphics environment. Computing in science \& engineering 9 , 90-95 (2007).

24. Punjani, A., Rubinstein, J.L., Fleet, D.J. \& Brubaker, M.A. cryoSPARC: algorithms for rapid unsupervised cryo-EM structure determination. Nature methods 14, 290-296 (2017).

25. Abraham, M.J. et al. GROMACS: High performance molecular simulations through multi-level parallelism from laptops to supercomputers. SoftwareX 1, 19-25 (2015).

26. Souza Junior, J.B. et al. Pair distribution function from electron diffraction in cryogenic electron microscopy: revealing glassy water structure. The journal of physical chemistry letters 11, 1564-1569 (2020).

27. Himes, B. \& Grigorieff, N. Cryo-TEM simulations of amorphous radiation-sensitive samples using multislice wave propagation. IUCrJ 8, 943-953 (2021).

28. Hall, R., Nogales, E. \& Glaeser, R. Accurate modeling of single-particle cryo-EM images quantitates the benefits expected from using Zernike phase contrast. Journal of structural biology 174, 468-475 (2011).

29. Brown, H.G., Pelz, P., Ophus, C. \& Ciston, J. A python based open-source multislice simulation package for transmission electron microscopy. Microscopy and Microanalysis 26, 2954-2956 (2020).

30. Egerton, R.F. Electron energy-loss spectroscopy in the electron microscope. (Springer Science \& Business Media, 2011).

31. Kremer, J.R., Mastronarde, D.N. \& McIntosh, J.R. Computer visualization of threedimensional image data using IMOD. Journal of structural biology 116, 71-76 (1996).

32. Scheres, S.H. RELION: implementation of a Bayesian approach to cryo-EM structure determination. Journal of structural biology 180, 519-530 (2012).

33. Asarnow, D., Palovcak, E., Cheng, Y. UCSF pyem v0.5, https://doi.org/10.5281/zenodo.3576630 (2019).

34. Pettersen, E.F. et al. UCSF: ChimeraX: Structure visualization for researchers, educators, and developers; . Protein Science 30, 70-82. 

(a)

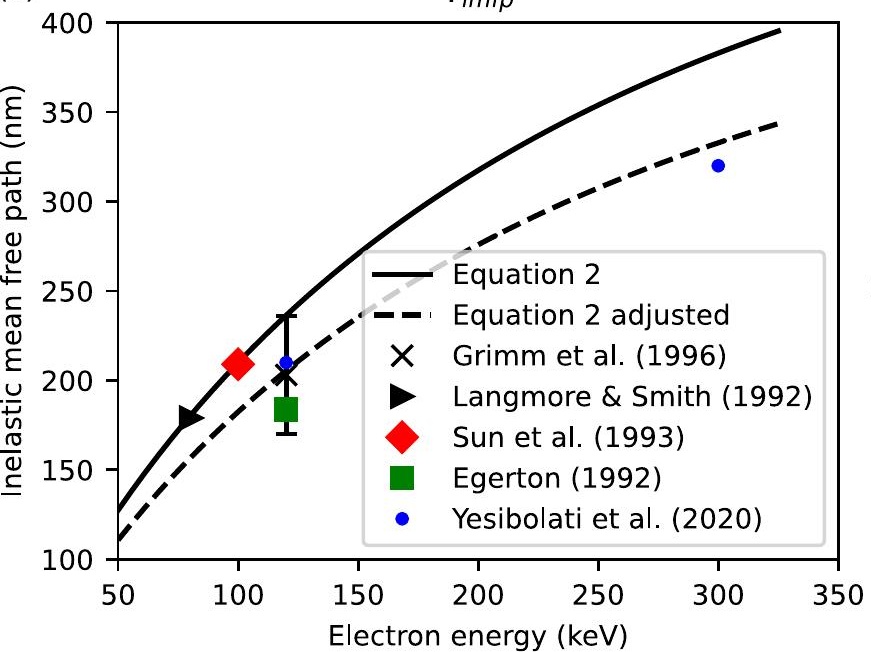

(b)

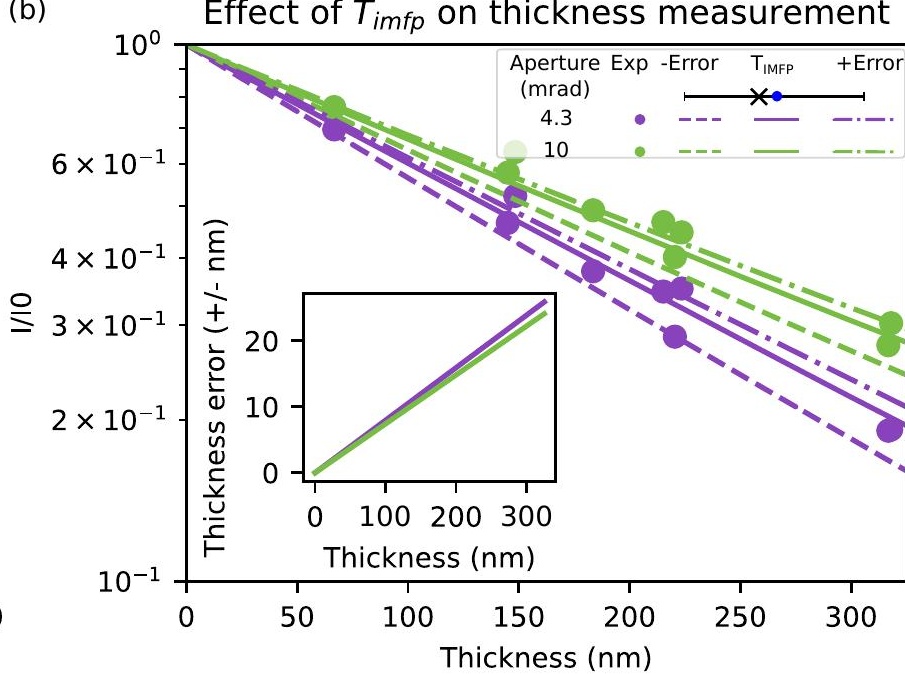



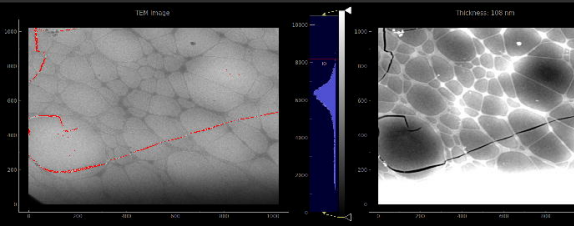

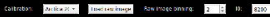


(d) Aperture limited

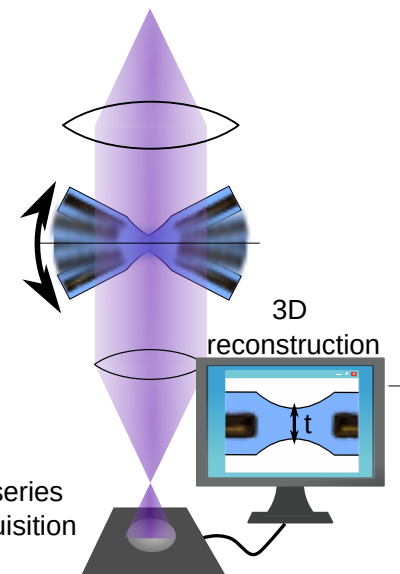

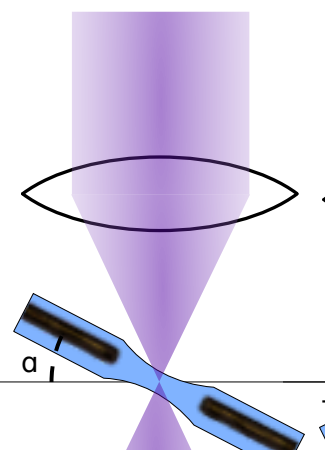

Hole burnt in specimen $a t+a$
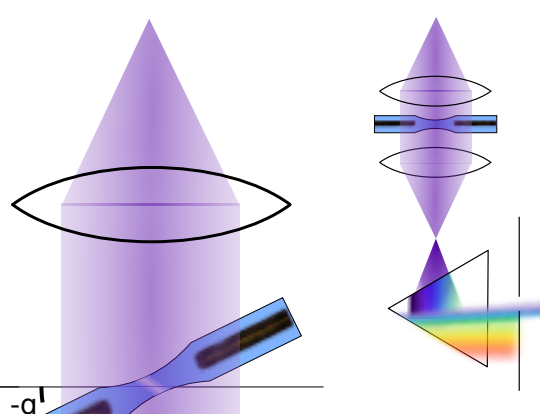

Ice thickness inferred from image measured at $-a$
Post-specimen energy filter

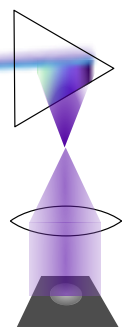

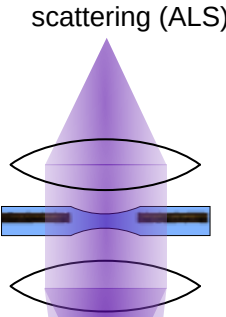

Objective aperture

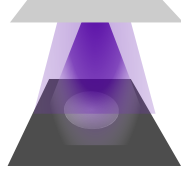


(a) Tomography

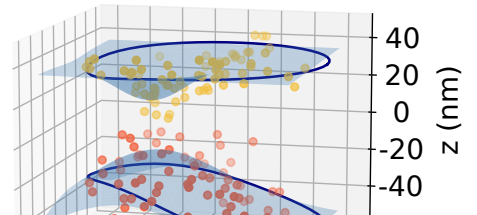

Top surface particles

Bottom surface particles

$0 \quad 400 \underset{x(\mathrm{~nm})}{800} 1200$

(b) Single hole thickness maps

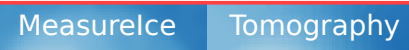

$0.5 \mu \mathrm{m}$

$0 \quad 20 \quad 40 \quad 60 \quad 80 \quad 100$
Ice thickness $(\mathrm{nm})$

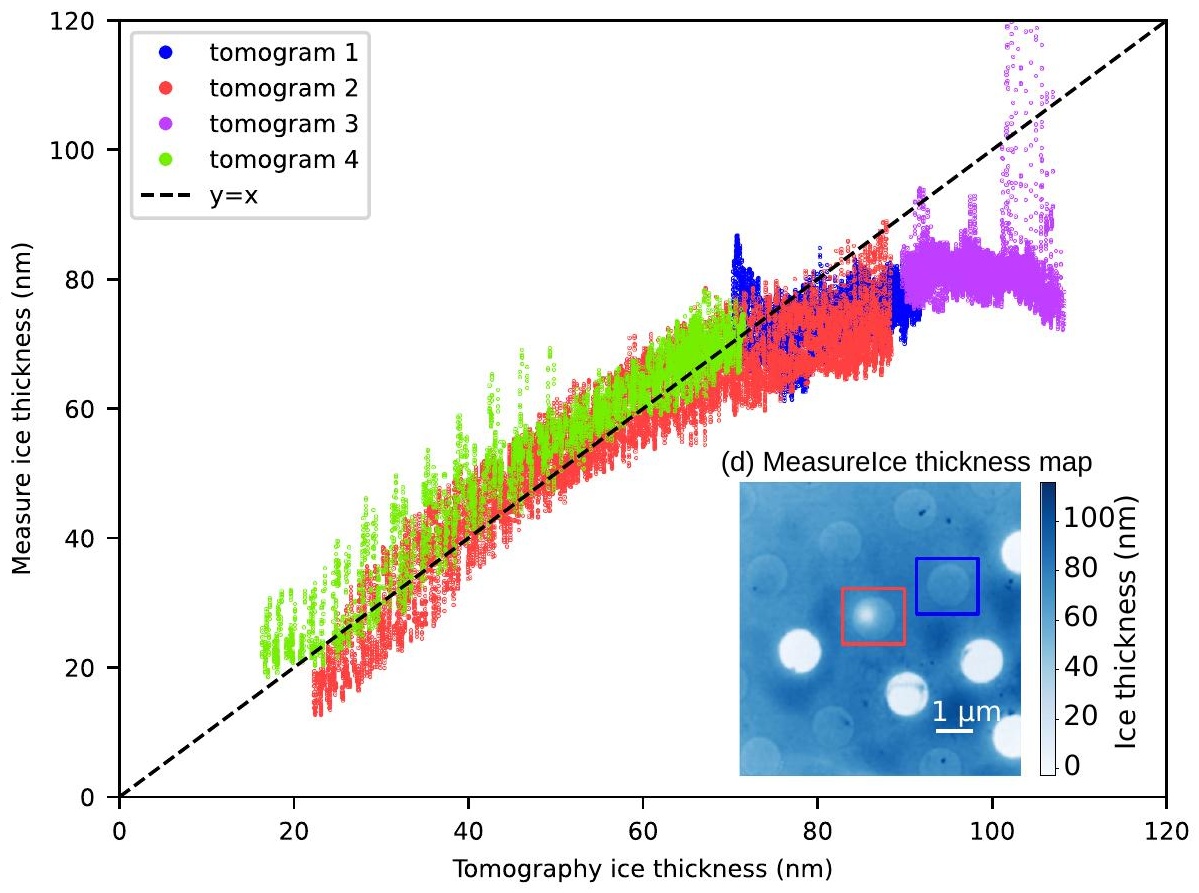

